\title{
A LEVEL SET FORMULATION FOR THE 3D INCOMPRESSIBLE EULER EQUATIONS*
}

\author{
JIAN DENG ${ }^{\dagger}$, THOMAS Y. HOU ${ }^{\ddagger}$, AND XINWEI YU§
}

\begin{abstract}
We explore a level set representation of vorticity in the study of the singularity problems for incompressible fluid models. This representation exists for all initial vorticity fields. We further apply it to study the 3D Lagrangian averaged Euler equations and the 3D Euler equations, and obtain new global existence conditions.
\end{abstract}

Key words. Level sets, Clebsch representation, 3D Euler, non-blowup

AMS subject classifications. 76B03, 35Q35

1. Introduction. It is well-known that the 3D incompressible Euler equations

$$
\begin{aligned}
u_{t}+u \cdot \nabla u & =-\nabla p \\
\nabla \cdot u & =0
\end{aligned}
$$

exhibit rich geometric structures. Here $u$ is velocity and $p$ is pressure. One way to explore these structures is through the so-called Clebsch variables ([7]), which represent the vorticity by two level set functions $\phi, \psi: \mathbb{R}^{3} \mapsto \mathbb{R}$ as follows.

$$
\omega=\nabla \phi \times \nabla \psi,
$$

where $\phi$ and $\psi$ are carried by the flow, that is

$$
\begin{aligned}
\phi_{t}+u \cdot \nabla \phi & =0, \\
\psi_{t}+u \cdot \nabla \psi & =0 .
\end{aligned}
$$

The above Clebsch representation has important applications in the study of the Hamiltonian structure of the ideal fluids. See e.g. Marsden-Weinstein [24].

Despite its usefulness, it turns out that many interesting vorticity fields do not admit Clebsch variables representation (1.3). For example, a necessary condition for (1.3) to hold in the whole space is

$$
\mathcal{H} \equiv \int_{\mathbb{R}^{3}} u \cdot \omega=0
$$

which implies that the topology of the vorticity vector field must be very simple. Even local existence of the Clebsch representation is only guaranteed at points where the vorticity does not vanish. It has been shown (Graham-Henyey [17]) that in general (1.3) can not hold around a point with zero vorticity. For more discussion on the properties of classical Clebsch variables and its various generalizations, see Constantin [12], Graham-Henyey [17], Marsden-Weinstein [24] and related references therein.

\footnotetext{
*Received August 24, 2005; accepted for publication March 28, 2006. This work was supported in part by NSF under the NSF FRG grant DMS-0353838 and ITR grant ACI-0204932.

${ }^{\dagger}$ Department of Mathematics, Fudan University, Shanghai, China (jdeng@fudan.edu.cn).

¥Applied and Comput. Math, Caltech, Pasadena, CA 91125, USA (hou@acm.caltech.edu).

$\S$ Department of Mathematics, UCLA, Box 951555, Los Angeles, CA 90095, USA (xinweiyu@ math.ucla.edu).
} 
Although much study has been done on Clebsch variables and other types of representations involving level set functions carried by the flow, so far few works have addressed the possibility of utilizing such representations in the study of singularity problems in fluid dynamics, for example, the singularity problems of the 3D incompressible Euler equations and the 3D Lagrangian averaged Euler equations. The only effort for the 3D Euler equations, to the authors' knowledge, is Constantin [9, 10, 11]. There a generalization of the Clebsch formula has been used to obtain local exsitence of the solutions to the 3D incompressible Euler equations. We remark that in a recent paper, Hou and $\mathrm{Li}[20]$ have explored the level set formulation in their study of the global existence of the 3D Lagrangian averaged Euler equations.

In this paper, we present a level set representation for the vorticity field which always exists in the whole space. This representation is closely related to the Weber formula and can be viewed as a special case of the magnetization variables. We show that new understanding of the singularity problems of the 3D Euler equations and 3D Lagrangian averaged Euler equations can be gained by application of this level set representation in the analysis of the vorticity growth.

The remaining of this paper is organized as follows. In Section 2, we will present a level set representation of the vorticity field, and study its properties. In Section 3, we apply this level set representation to study the 3D Lagrangian averaged Euler and 3D Euler singularity problems. Finally, in Section 4, we will make a few concluding remarks.

2. A Level Set Formulation. First we review the theory of classical Clebsch variables. The Clebsch variables are two level set functions $\phi, \psi: \mathbb{R}^{3} \mapsto \mathbb{R}$ that are carried by the flow, i.e.,

$$
\begin{aligned}
\phi_{t}+u \cdot \nabla \phi & =0, \\
\psi_{t}+u \cdot \nabla \psi & =0 .
\end{aligned}
$$

Using these two level sets, the velocity $u$ can by represented as

$$
u=\phi \nabla \psi+\nabla n
$$

where $n$ is chosen to enforce the incompressibility condition: $\nabla \cdot u=0$. In this setting, the vorticity $\omega=\nabla \times u$ can be written as

$$
\omega=\nabla \phi \times \nabla \psi .
$$

(2.4) is more geometrical than (2.3) in the sense that from (2.4) one clearly sees that vortex lines are just the intersection curves of the level set surfaces of $\phi$ and $\psi$, and the stretching/thinning of vortex tubes can be visualized as the collapsing of these surfaces.

It has been found that the above Clebsch representation only holds for a very limited class of flows, as we have mentioned in Section 1. We quickly review major properties of classical Clebsch variables, including its limitations, here.

- If $\omega=\nabla \phi \times \nabla \psi$ at one time $s$, then $\omega=\nabla \phi \times \psi$ for all times $t>s$. Therefore to show that a particular flow has a Clebsch representation, we only need to check it at the initial time $t=0$.

- If $\omega=\nabla \phi \times \nabla \psi$, then the helicity $\mathcal{H} \equiv \int u \cdot \omega=0$. To prove this, one just 
need to notice that

$$
\begin{aligned}
u \cdot \omega & =(\phi \nabla \psi+\nabla n) \cdot(\nabla \phi \times \nabla \psi) \\
& =\nabla n \cdot \omega \\
& =\nabla \cdot(n \omega)
\end{aligned}
$$

where we have used $\nabla \cdot \omega=0$. Note that vanishing helicity requires the flow to be simple. For example, there cannot be two closed vortex lines that form linked rings.

- If $\omega=\nabla \phi \times \nabla \psi$ in a neighborhood of a point $x_{0}$ where the vorticity vanishes, then $\operatorname{det}\left[\nabla \omega\left(x_{0}\right)\right]=0$ (Graham-Henyey [17]). To prove this, just compute

$$
\begin{aligned}
(\nabla \omega)_{i j} & =\partial_{j}\left(\omega_{i}\right) \\
& =\partial_{j}\left(\varepsilon_{i l m} \partial_{l} \phi \partial_{m} \psi\right) \\
& =\varepsilon_{i l m}\left(\partial_{j} \partial_{l} \phi\right) \partial_{m} \psi+\varepsilon_{i l m} \partial_{l} \phi \partial_{j} \partial_{m} \psi
\end{aligned}
$$

where $\varepsilon_{i l m}=1$ when $(i, l, m)$ is an even permutation of $(1,2,3),=-1$ when $(i, l, m)$ is an odd permutation, and 0 otherwise. Now notice that when neither $\nabla \phi$ nor $\nabla \psi$ vanishes at $x_{0}, \omega\left(x_{0}\right)=0$ implies $\nabla \phi \| \nabla \psi$. Therefore $\nabla \omega \cdot \xi=0$ for any $\xi \| \nabla \phi$, since $\varepsilon_{i l m} \partial_{m} \psi \xi_{i}=(\nabla \psi \times \xi)_{l}=0$ and for the same reason $\varepsilon_{i l m} \partial_{l} \phi \xi_{i}=0$.

- If $\omega \neq 0$ at some $x_{0}$, then $\omega=\nabla \phi \times \nabla \psi$ for some $\phi$ and $\psi$ in a neighborhood of $x_{0}$. The proof of this is a simple application of first order PDE theory. For example, since $\omega\left(x_{0}\right) \neq 0$, we can find a constant vector $v$ such that $v \times \omega \neq 0$ in a small neighborhood. Thus the equation

$$
(v \times \omega) \cdot \nabla \phi=0
$$

is well-posed in a neighborhood of $x_{0}$ and therefore $\phi$ is obtained. Now we further solve

$$
\nabla \phi \times \nabla \psi=\omega
$$

in a (maybe smaller) neighborhood of $x_{0}$ to get $\psi$.

Since many interesting flows can not be represented by Clebsch variables, it is important to find other level set formulations that can be used to represent more general fluid flows. Indeed, many such level set representations have been studied, for example the Weber formula (Weber [26]), the magnetization variables (Buttke-Chorin [3]) and the Eulerian Lagrangian formulation (Constantin [9]).

In this paper, we present a special form of level set representation that exists for all flows and show that it can be used to improve our understanding of the geometry of possible blow-up scenario of the 3D Euler and the averaged Euler equations. For convenience, we will refer to this representation as Generalized Clebsch Variables.

Definition 2.1. (Generalized Clebsch Variables). The Generalized Clebsch Variables are two triplets of real functions $\Phi=\left(\phi_{1}, \phi_{2}, \phi_{3}\right)$ and $\mathbf{U}=$ $\left(U_{1}(\Phi), U_{2}(\Phi), U_{3}(\Phi)\right)$, such that the vorticity vector field $\omega$ can be represented in the following way:

$$
\omega=\sum_{k=1}^{3} \nabla U_{k} \times \nabla \phi_{k} .
$$


REMARK 2.2. The theory of magnetization variables (Buttke-Chorin [3], we follow Smith-Porkoný [25] here) shows the existence of a "magnetization variable" $\mathbf{m}$ such that $\omega=\nabla \times \mathbf{m}$, and

$$
\mathbf{m}=\sum_{i=1}^{R} \phi_{i} \nabla \psi_{i}
$$

for all time if (2.14) holds at $t=0$, and each $\phi_{i}$ and $\psi_{i}$ are carried by the flow:

$$
\left(\phi_{i}\right)_{t}+u \cdot \nabla \phi_{i}=0, \quad\left(\psi_{i}\right)_{t}+u \cdot \nabla \psi_{i}=0
$$

Taking $\nabla \times$ of $\mathbf{m}$, we immediately see that

$$
\omega=\sum_{i=1}^{R} \nabla \phi_{i} \times \nabla \psi_{i} .
$$

Therefore the Generalized Clebsch Variables representation (2.13) can be viewed as a special case of (2.14) when $R=3$ and $\psi_{i}$ depends on $\left(\phi_{1}, \phi_{2}, \phi_{3}\right)$ only. It turns out that this special case can be conveniently applied to the study of singularity problems, as we will see in Section 3.

Now we summarize some properties of the Generalized Clebsch Variables representation (2.13).

- The representation (2.13) remains true at later times if it is true at $t=0$ and all $\phi_{i}, i=1,2,3$ are transported by the flow. To see this, we only need to notice that since $U_{k}=U_{k}(\Phi), U_{k}$ is also transported by the flow. Therefore each $\nabla U_{k} \times \nabla \phi_{k}$ is a Clebsch representation of some divergence free vector field. Thus

$$
\omega=\sum_{k=1}^{3} \nabla U_{k} \times \nabla \phi_{k}
$$

holds at later times due to the same property of the classical Clebsch variables. Also note that this property is not confined with ideal fluids. For example, one can easily show that it also holds for the Lagrangian averaged Euler flow.

- The representation (2.13) exists for all initial vorticity fields. To see this, we take $\phi_{k}=x_{k}$ to be the coordinate functions, and take $U_{k}=u_{k}$ to be the $k$ th component of the velocity vector. Then it is clear that

$$
\omega=\sum_{k=1}^{3} \nabla U_{k} \times \nabla \phi_{k} .
$$

- When $\omega_{0}$ is compactly supported, it is easy to see that we can modify the initial $\Phi=\left(\phi_{1}, \phi_{2}, \phi_{3}\right)$ and $\mathbf{U}=\left(U_{1}, U_{2}, U_{3}\right)$ outside the support of $\omega_{0}$ such that each $\phi_{k}$ and $U_{k}$ are compactly supported, consequently bounded. Since they are just carried by the flow, we see that $\phi_{k}$ and $U_{k}$ are uniformly bounded in time. 
Before ending this section, we briefly address the relation between our generalized Clebsch variables and the Weber formula (Weber [26], we follow the presentation in Constantin [12] here). The Weber formula is given as follows:

$$
\begin{aligned}
\Phi_{t}+u \cdot \nabla \Phi & =0 \\
U_{t}+u \cdot \nabla U & =0 \\
u & =W[\Phi, U]
\end{aligned}
$$

where $\Phi=x$ and $U=u$ at $t=0 . W$ is defiend as

$$
W[\Phi, U]=\mathbf{P}\left\{(\nabla \Phi)^{*} \cdot U\right\}
$$

with $\mathbf{P}$ being the projection onto divergence free vector fields. We now show that the Weber formula (2.19)-(2.21) is equivalent to the Generalized Clebsch Variables representation

$$
\omega=\sum_{k=1}^{3} \nabla U_{k} \times \nabla \phi_{k}
$$

with $\Phi=\left(\phi_{1}, \phi_{2}, \phi_{3}\right)$ and $\mathbf{U}=\left(U_{1}, U_{2}, U_{3}\right)$.

Proof. We only need to calculate $\nabla \times u$ from the Weber formula. We have

$$
\begin{aligned}
\nabla \times u & =\nabla \times\left[\mathbf{P}\left\{(\nabla \Phi)^{*} \cdot U\right\}\right] \\
& =\nabla \times\left\{(\nabla \Phi)^{*} \cdot U\right\} \\
& =\sum_{k=1}^{3} \nabla U_{k} \times \nabla \phi_{k}
\end{aligned}
$$

after straightforward calculation of its $i$-th component

$$
\begin{aligned}
(\nabla \times u)_{i} & =\varepsilon_{i l m} \partial_{l}\left[\left(\partial_{m} \Phi_{k}\right) U_{k}\right] \\
& =\varepsilon_{i l m}\left(\partial_{l} \partial_{m} \Phi_{k}\right) U_{k}+\varepsilon_{i l m}\left(\partial_{l} U_{k}\right)\left(\partial_{m} \Phi_{k}\right) \\
& =0+\left(\sum_{k=1}^{3} \nabla U_{k} \times \nabla \Phi_{k}\right)_{i},
\end{aligned}
$$

where $\varepsilon_{i l m}=1$ when $(i, l, m)$ is an even permutation of $(1,2,3),=-1$ when $(i, l, m)$ is an odd permutation, and 0 otherwise. $\square$

We see that the Generalized Clebsch Variables representation is the same as the Weber formula when we take $\Phi=\left(\phi_{1}, \phi_{2}, \phi_{3}\right)$ to be the Lagrangian Cartesian coordinate system. However, the Generalized Clebsch Variables formula (2.13) allows a wider choice of the level set functions. This makes it easier to apply the Generalized Clebsch Variables representation to singularity problems, as we will see in Section 3.

3. Application to Singularity Problems. In the section, we present applications of the Generalized Clebsch Variables in the study of singularity problems for certain incompressible fluids. The flows considered here are the 3D Lagrangian averaged Euler equations and the 3D Euler equations. 
3.1. Applications to 3D Lagrangian averaged Euler equations. The Lagrangian averaged Euler equations, recently developed in Holm-Marsden-Ratiu $[18,19]$, try to catch the averaged behavior of the 3D incompressible Euler flow, and have been used as a turbulence closure model (Chen et. al. [5]). The theoretical and computational aspects of the Lagrangian averaged Euler equations has been studied by various authors $([6,18,19,20,22,23])$. However, the global existence of the classical solution to the Lagrangian averaged Euler equations still have not been established. In Hou-Li [20], a sufficient condition has been given for initial values admitting a Clebsch representation. Here we will generalize their result to initial values admitting a Generalized Clebsch Variables representation.

We first recall the formulation of the Lagrangian averaged Euler equations. We follow the notations in Hou-Li [20].

The main equation reads

$$
u_{t}+u_{\alpha} \cdot \nabla u+\left(\nabla u_{\alpha}\right)^{T} \cdot u=-\nabla p,
$$

where $u_{\alpha} \equiv\left(1-\alpha^{2} \triangle\right)^{-1} u$, and $u$ is required to be divergence free. Written in vorticity form, (3.1) becomes

$$
\omega_{t}+u_{\alpha} \cdot \nabla \omega=\nabla u_{\alpha} \cdot \omega
$$

where the vorticity $\omega \equiv \nabla \times u$.

Comparing with the vorticity form of the 3D Euler equations,

$$
\begin{aligned}
\omega_{t}+u \cdot \nabla \omega & =\nabla u \cdot \omega \\
\nabla \times u & =\omega
\end{aligned}
$$

we see that the vorticity is still convected as a two form, only the velocity field has been regularized.

In Hou-Li [20], the following existence result is proved.

For any function $\phi$, we define

$$
\|\phi\|_{T V_{x_{1}}}=\sup _{x_{2}, x_{3}} \int_{-\infty}^{\infty}\left|\frac{\partial}{\partial x_{1}} \phi\left(x_{1}, x_{2}, x_{3}\right)\right| d x_{1},
$$

and the TV norm

$$
\|\phi\|_{T V}=\sum_{i=1}^{3}\|\phi\|_{T V_{x_{i}}},
$$

which measures the oscillation of $\phi$ along coordinate directions.

Now assume that the initial vorticity has the form $\omega(0, x)=\omega_{0}\left(\phi_{0}, \psi_{0}\right) \nabla \phi_{0} \times$ $\nabla \psi_{0}$ with $\omega_{0}, \phi_{0}$ and $\psi_{0}$ being smooth and bounded. Further assume that $\phi, \psi$ are carried by the flow with initial values $\phi_{0}, \psi_{0}$. With these assumptions, the Lagrangian averaged 3D Euler equations have a unique smooth solution up to $T$ as long as either $\int_{0}^{T}\|\phi\|_{T V} d t<\infty$ or $\int_{0}^{T}\|\psi\|_{T V} d t<\infty$. Furthermore, the following estimate holds

$$
\|\omega(t)\|_{H^{m}} \leq C(T)\|\omega(0)\|_{H^{m}}, \quad 0 \leq t \leq T
$$

for $m>5 / 2$.

Now we present our generalization of this theorem. 
THEOREM 3.1. For any initial vorticity field that is bounded and with compact support, take $\mathbf{U}=\left(U_{1}, U_{2}, U_{3}\right)$ and $\Phi=\left(\phi_{1}, \phi_{2}, \phi_{3}\right)$ to be its Generalized Clebsch Variables representation. Then the Lagrangian averaged Euler equations have a unique smooth solution up to $T$ as long as $\int_{0}^{T}\left\|\phi_{i}\right\|_{T V} d t<\infty$ for any two $i \in\{1,2,3\}$, and $\frac{\partial U_{k}}{\partial \phi_{k}}=0$ for the remaining index $k$.

Furthermore, the energy estimate

$$
\|\omega(t)\|_{H^{m}} \leq C(T)\|\omega(0)\|_{H^{m}}, \quad 0 \leq t \leq T
$$

holds for $m>5 / 2$.

REMARK 3.2. It is worth mentioning that, since $U$ depends on $\Phi$ only, the assumption $\frac{\partial U_{k}}{\partial \phi_{k}}=0$ is an assumption on the initial value, and does not assume anything for the later evolution of the flow. Therefore to apply this theorem, one just need to choose initial vorticity such that $\frac{\partial U_{k}}{\partial \phi_{k}}=0$. We would like to further point out that many interesting flows satisfy this constraint. For example, if we take $\Phi=(r, z, \theta)$ and take the initial flow to be axisymmetric, then automatically $\frac{\partial U_{3}}{\partial \theta}=0$.

Proof. We first recall the main steps of the proof by Hou-Li for the case $\omega=$ $\nabla \phi \times \nabla \psi$, with the assumption that $\|\psi\|_{T V}<\infty$.

Since $u_{\alpha}=\left(1-\alpha^{2} \triangle\right)^{-1} \nabla \times(-\triangle)^{-1} \omega$, it is easy to derive that

$$
\nabla u_{\alpha}(0)=\int_{\mathbb{R}^{3}} \nabla B(y) \times(\phi(y) \nabla \psi(y)) d y,
$$

where for $\nabla B(y)$ one has the estimate

$$
|\nabla B(y)| \leq \frac{C_{\alpha}}{|y|^{2}\left(1+|y|^{1 / 4}\right)}
$$

Let $B_{\varepsilon}$ denote the ball centered at the origin with radius $\varepsilon<1$. Let $p>3, q$ be such that $\frac{1}{p}+\frac{1}{q}=1$, and further denote $r=\frac{3-2 q}{q}$. Now for $i \in\{1,2,3\}$, we denote by $y^{\prime}$ the remaining two dimensional vector excluding $y_{i}$. Then

$$
\begin{aligned}
\left|\nabla u_{\alpha}(0)\right| & =\left|\int_{B_{\varepsilon}}+\int_{|y| \geq \varepsilon} \nabla B(y) \times(\phi(y) \nabla \psi(y)) d y\right| \\
\leq & \|\phi\|_{\infty}\left(\varepsilon^{r}\|\nabla \psi\|_{L^{p}}+\sum_{i=1}^{3} \int_{\left|y^{\prime}\right|^{2}+\left|y_{i}\right|^{2} \geq \varepsilon^{2}} \frac{\int\left|\frac{\partial \psi}{\partial y_{i}}\right| d y_{i}}{\left(\left|y_{i}\right|^{2}+\left|y^{\prime}\right|^{2}\right)\left(1+|y|^{1 / 4}\right)} d y^{\prime}\right) \\
\leq & \|\phi\|_{\infty}\left(\varepsilon^{r}\|\nabla \psi\|_{L^{p}}+\|\psi\|_{T V} \int_{\mathbb{R}^{2}} \frac{d y^{\prime}}{\left(\varepsilon^{2}+\left|y^{\prime}\right|^{2}\right)\left(1+|y|^{1 / 4}\right)}+\right. \\
& \left.\|\| \phi \|_{T V} \int_{\left|y^{\prime}\right|^{2} \geq \varepsilon} \frac{d y^{\prime}}{\left|y^{\prime}\right|^{2}\left(1+\left|y^{\prime}\right|^{1 / 4}\right)}\right) \\
& \left.\left\|\varepsilon^{r}\right\| \nabla \psi\left\|_{L^{p}}+\right\| \psi \|_{T V} \log \frac{1}{\varepsilon}\right) .
\end{aligned}
$$


where the Hölder inequality is used in the inner part estimate. By setting $\varepsilon^{r}\left(1+\|\nabla \psi\|_{L^{p}}\right)=1$, we obtain

$$
\left|\nabla u_{\alpha}(0)\right| \leq C\left(1+\|\psi\|_{T V} \log \left(\|\nabla \psi\|_{L^{p}}+1\right)\right) .
$$

Now recall that

$$
\psi_{t}+u_{\alpha} \cdot \nabla \psi=0
$$

Standard technique gives

$$
\begin{aligned}
\frac{\partial}{\partial t}\|\nabla \psi\|_{L^{p}} & \leq\left\|\nabla u_{\alpha}\right\|_{\infty}\|\nabla \psi\|_{L^{p}} \\
& \leq C\left(1+\|\psi\|_{T V} \log \left(\|\nabla \psi\|_{L^{p}}+1\right)\right)\|\nabla \psi\|_{L^{p}} .
\end{aligned}
$$

This implies

$$
\|\nabla \psi\|_{L^{p}} \leq C(T)
$$

due to the Gronwall inequality and the assumption that $\int_{0}^{T}\|\nabla \psi\|_{T V}<\infty$. Therefore we obtain

$$
\int_{0}^{T}\left\|\nabla u_{\alpha}\right\|_{\infty} d t \leq C \int_{0}^{T}\|\psi\|_{T V} d t \leq C(T) .
$$

Now it is standard to get the well-posedness of the solution.

Now we turn to our generalization. Since $\omega$ has compact support, we know that we can take $U_{i}$ 's to be uniformly bounded. Recall that

$$
\omega=\sum_{k} \nabla U_{k} \times \nabla \phi_{k} .
$$

Without loss of generality, we assume that the TV norm of $\phi_{1}, \phi_{2}$ are integrable in time, and $\frac{\partial U_{3}}{\partial \phi_{3}}=0$. Using a standard argument, we only need to show

$$
\int_{0}^{T}\left\|\nabla u_{\alpha}\right\|_{\infty} d t<\infty
$$

Since $\nabla u_{\alpha}$ and $\omega$ are related by the linear operator $\nabla\left(1-\alpha^{2} \triangle\right)^{-1} \nabla \times(-\triangle)^{-1}$, it is enough to show the boundedness of $\int_{0}^{T}\left\|\nabla u_{\alpha}\right\|_{\infty} d t$ when $\omega$ is given by $\nabla U_{i} \times \nabla \phi_{i}$ for each $i=1,2,3$. For $i=1,2$, the same argument used by Hou-Li works after replacing $\psi$ by $\phi_{i}$ and $\phi$ by $U_{i}$. For $i=3$, note that

$$
\nabla U_{3} \times \nabla \phi_{3}=\nabla \times\left(\phi_{3} \nabla U_{3}\right) .
$$

Since $\frac{\partial U_{3}}{\partial \phi_{3}}=0, \nabla U_{3}=\frac{\partial U_{3}}{\partial \phi_{1}} \nabla \phi_{1}+\frac{\partial U_{3}}{\partial \phi_{2}} \nabla \phi_{2}$. Therefore the $T V$ norm of $U_{3}$ is integrable in time due to the boundedness of $\frac{\partial U_{3}}{\partial \phi_{1}}$ and $\frac{\partial U_{3}}{\partial \phi_{2}}$. Now replacing $\psi$ by $U_{3}$ in the argument by Hou-Li, we see that the key estimates (3.10) and (3.14) still hold since

$$
\int\left|\frac{\partial U_{1}}{\partial \phi_{1}} \frac{\partial \phi_{1}}{\partial y_{i}}+\frac{\partial U_{2}}{\partial \phi_{2}} \frac{\partial \phi_{2}}{\partial y_{i}}\right| \leq C\left[\int\left|\frac{\partial \phi_{1}}{\partial y_{i}}\right| d y_{i}+\int\left|\frac{\partial \phi_{2}}{\partial y_{i}}\right| d y_{i}\right]
$$

and

$$
\left\|\frac{\partial U_{1}}{\partial \phi_{1}} \nabla \phi_{1}+\frac{\partial U_{2}}{\partial \phi_{2}} \nabla \phi_{2}\right\|_{L^{p}} \leq C\left(\left\|\nabla \phi_{1}\right\|_{L^{p}}+\left\|\nabla \phi_{2}\right\|_{L^{p}}\right) .
$$

Thus we end the proof of the theorem. $\square$ 
3.2. Applications to $3 \mathrm{D}$ Euler equations. The singularity problem of the $3 \mathrm{D}$ incompressible Euler equations is a longstanding open problem. Much effort has been made to show the global existence under various assumptions, see Majda-Bertozzi [21] for a good summary of classical results. There are also some recent results deepening the understanding of the Euler dynamics, see e.g. Babin-Mahalov-Nicolaenko [1], Caflisch [4], Constantin-Fefferman-Majda [13], Cordoba-Fefferman [14], Deng-Hou$\mathrm{Yu}[15,16]$. In this subsection, we will show how the Generalized Clebsch Variables may be used to study this problem.

The 3D Euler equations read

$$
\begin{aligned}
u_{t}+u \cdot \nabla u & =-\nabla p \\
\nabla \cdot u & =0 .
\end{aligned}
$$

In 1984, Beale-Kato-Majda [2] showed that as long as

$$
\int_{0}^{T}\|\omega\|_{\infty} d t<\infty
$$

where $\omega \equiv \nabla \times u$ is the vorticity, the solution to the 3D Euler equations remains classical, or in other words, no blow-up would occur up to time T. In 1994, Constantin [8] shows that the evolution of $|\omega|$ is governed by the following equation

$$
D_{t}|\omega| \equiv|\omega|_{t}+u \cdot \nabla|\omega|=\alpha(x, t)|\omega|,
$$

where the stretching factor is defined as (we make the dependence on $t$ implicit)

$$
\alpha(x, t) \equiv \frac{3}{4 \pi} p \cdot v \cdot \int \frac{(\hat{y} \cdot \xi(x)) \operatorname{det}(\hat{y}, \xi(x+y), \xi(x))}{|y|^{3}}|\omega(x+y)| d y,
$$

where $\hat{y} \equiv y /|y|$ and $\xi \equiv \omega /|\omega|$ is the unit vorticity vector. Thus according to the BKM criterion (3.23), it suffices to show that

$$
\int_{0}^{T} \alpha(x, t) d t<\infty
$$

to guarantee the existence of classical solutions. In Constantin-Fefferman-Majda [13], $|\alpha(x, t)|$ is carefully estimated, and the following result is proved.

Assume that there exists $\rho>0$ and $0<r \leq \frac{\rho}{2}$ such that the following conditions are satisfied for a set $W_{0}$.

Firstly, for every $q \in W_{0}^{*} \equiv\left\{q \in W_{0} ;\left|\omega_{0}(q)\right| \neq 0\right\}$, the function $\xi(\cdot, t)$ has a Lipschitz extension (denoted by the same letter) to the Euclidean ball of radius $4 \rho$ centered at $X(q, t)$, denoted as $B_{4 \rho}(X(q, t))$, and

$$
M=\sup _{q \in W_{0}^{*}} \int_{0}^{T}\|\nabla \xi\|_{L^{\infty}\left(B_{4 \rho}(X(q, t))\right)}^{2} d t<\infty .
$$

Secondly,

$$
\sup _{B_{4 \rho}\left(W_{t}\right)}|\omega(x, t)| \leq m \sup _{B_{r}\left(W_{t}\right)}|\omega(x, t)|
$$

holds for all $t \in[0, T)$ with $m \geq 0$ constant. Here

$$
W_{t} \equiv X\left(W_{0}, t\right)
$$


And thirdly,

$$
\sup _{B_{4 \rho}\left(W_{t}\right)}|u|<U
$$

Then there will be no singularity in the region $W_{t}$ up to $T$.

REMARK 3.3. Assumptions (3.27) and (3.30) put two constraints on the flow. The intuition is that, when the flow behaves nicely, or more specifically, the vorticity vectors are aligned to some extent and the velocity remains bounded, then no blow-up can occur. (3.28) is a technical assumption.

Now we state our result. Assume that the vorticity has been represented by the Generalized Clebsch Variables,

$$
\omega=\sum_{k=1}^{3} \nabla U_{k} \times \nabla \phi_{k} .
$$

Then we have the following theorem.

THEOREM 3.4. We assume that (3.27) and (3.28) hold. If furthermore

1. for all $t \in[0, T)$,

$$
\sup _{B_{4 \rho}\left(W_{t}\right)}\left|\nabla \phi_{i}\right| \leq U
$$

for some constant $U$, for two i's in $\{1,2,3\}$.

2. $U_{k}$ is independent of $\phi_{k}$ for the remaining index $k$, i.e.,

$$
\frac{\partial U_{k}}{\partial \phi_{k}}=0
$$

Then there exists $\tau>0$ and $\Gamma$ such that

$$
\sup _{B_{r}\left(W_{t}\right)}|\omega(x, t)| \leq \Gamma \sup _{B_{\rho}\left(W_{t_{0}}\right)}\left|\omega\left(x, t_{0}\right)\right|,
$$

therefore the solution exists beyond $T$.

REMARK 3.5. Before we present the proof, we would like to make a few remarks.

1. As we have pointed out in Remark 3.2, the constraint (3.33) is on the initial value only, and does not require the flow to have any special properties at later times. Also, many interesting flows indeed satisfy (3.33), for example the axisymmetric flow, for which the global existence is stll unsettled.

2. The major difference between Theorem 3.4 and the main theorem in Constantin-Fefferman-Majda [13] is that a physical condition

$$
\sup _{B_{4 \rho}\left(W_{t}\right)}|u|<U .
$$

is replaced by a geometrical condition

$$
\sup _{B_{4 \rho}\left(W_{t}\right)}\left|\nabla \phi_{i}\right| \leq U
$$


The major gain of the latter is that one can take $\phi_{i}$ to indicate geometrical properties of the (possible) singularity. For example, if we take $\left(\phi_{1}, \phi_{2}, \phi_{3}\right)$ to be the initial coordinate functions, then the conditions sup $\left|\nabla \phi_{1}\right|, \sup \left|\nabla \phi_{2}\right|<$ $U$ basically confine our consideration to the following special criterion of singularity formation: two level set surfaces of $\phi_{3}$ collapsing with each other.

3 . In the case of axisymmetric flows, we can take $\left(\phi_{1}, \phi_{2}, \phi_{3}\right)=(r, z, \theta)$. Then automatically (3.33) is satisfied. Thus Theorem 3.4 excludes the following type of singularity: the vortex lines stretch wildly in the $\theta$ direction, while the changes in the $r, z$ directions are mild. This profile of singularity is impossible since, in this case, the vorticity direction $\xi$ would be varying slowly, and the Lagrangian coordinate functions $R, Z$ (viewed as functions of $r, z, \theta$ ) also vary mildly in space, and therefore the assumptions in the theorem hold. This insight is new and may help checking numerical simulations searching for axisymmetric singularities.

Proof. The proof follows closely the one in Constantin-Fefferman-Majda [13]. We decompose

$$
\alpha(x)=\alpha_{\text {in }}(x)+\alpha_{\text {out }}(x)
$$

where

$$
\alpha_{\in}(x)=p \cdot v \cdot \int \chi\left(\frac{|y|}{\rho}\right)(\hat{y} \cdot \xi(x)) \operatorname{det}(\hat{y}, \xi(x+y), \xi(x))|\omega(x+y)| \frac{d y}{|y|^{3}}
$$

and

$$
\alpha_{\text {out }}(x)=\int\left(1-\chi\left(\frac{|y|}{\rho}\right)\right)(\hat{y} \cdot \xi(x)) \operatorname{det}(\hat{y}, \xi(x+y), \xi(x))|\omega(x+y)| \frac{d y}{|y|^{3}}
$$

with $\chi(r)$ being a smooth non-negative function satisfying $\chi(r)=1$ for $r \leq 1 / 2$ and 0 for $r \geq 1$. Now using $\xi(x+y)|\omega(x+y)|=\omega(x+y)=\nabla \times u(x+y)$, we can do integration by parts for $\alpha_{\text {out }}$ and get

$$
\left|\alpha_{\text {out }}(x)\right| \leq C \rho^{-5 / 2}\left\|u_{0}\right\|_{L^{2}}
$$

after applying Cauchy-Schwarz inequality. Therefore $\left|\alpha_{\text {out }}\right|$ stays bounded.

To estimate $\alpha_{i n}$, we denote

$$
G_{\rho}(x)=\sup _{|y| \leq \rho}|\nabla \xi(x+y)| .
$$

Since $\operatorname{det}(\hat{y}, \xi(x+y), \xi(x)) \leq G_{\rho}(x)|y|$, we have

$$
\left|\alpha_{i n}(x)\right| \leq G_{\rho}(x) I(x)
$$

with

$$
I(x) \equiv \int \chi\left(\frac{|y|}{\rho}\right)|\omega(x+y)| \frac{d y}{|y|^{2}} .
$$

Next we split $I=I_{1}+I_{2}$, where

$$
I_{1}(x)=\int \chi\left(\frac{|y|}{\delta}\right) \chi\left(\frac{|y|}{\rho}\right)|\omega(x+y)| \frac{d y}{|y|^{2}}
$$


and

$$
I_{2}(x)=\int\left[1-\chi\left(\frac{|y|}{\delta}\right)\right] \chi\left(\frac{|y|}{\rho}\right)|\omega(x+y)| \frac{d y}{|y|^{2}}
$$

with $\delta \leq \rho / 2$ to be fixed. Then using polar coordinates, we easily see

$$
\left|I_{1}(x)\right| \leq C \delta \Omega_{\delta}(x)
$$

where

$$
\Omega_{\delta}(x)=\sup _{|y| \leq \delta}|\omega(x+y)| .
$$

To estimate $I_{2}$, we replace $|\omega(x+y)|$ by $\xi(x+y) \cdot \omega(x+y)$. Note that $\nabla U_{i} \times \nabla \phi_{i}=$ $\nabla \times\left(U_{i} \nabla \phi_{i}\right)$ for $i=1,2$, and

$$
\begin{aligned}
\nabla U_{3} \times \nabla \phi_{3} & =\nabla \times\left(\phi_{3} \nabla U_{3}\right) \\
& =\nabla \times\left[\phi_{3}\left(\frac{\partial U_{3}}{\partial \phi_{1}} \nabla \phi_{1}+\frac{\partial U_{3}}{\partial \phi_{2}} \nabla \phi_{2}\right)\right]
\end{aligned}
$$

since by assumption $U_{3}$ is independent of $\phi_{3}$. Therefore by our assumption we see that

$$
\omega=\nabla \times \tilde{u}
$$

for some function $\tilde{u}$ satisfying the bound

$$
\sup _{B_{4 \rho}\left(W_{t}\right)}|\tilde{u}|<\tilde{U}
$$

for any $t \in[0, T)$, for some constant $\tilde{U}$. Therefore

$$
\begin{aligned}
I_{2}(x) & =\int \tilde{u}(x+y)\left\{\nabla \times\left[\frac{\xi(x+y)}{|y|^{2}} \chi\left(\frac{|y|}{\rho}\right)\left(1-\chi\left(\frac{|y|}{\delta}\right)\right)\right]\right\} d y \\
& =A+B+C+D
\end{aligned}
$$

where the four terms comes from applying $\nabla \times$ to the four terms in [.]. Now by the same estimates as in Constantin-Fefferman-Majda, we have

$$
\begin{aligned}
|A| & \leq C \rho \tilde{U} G_{\rho}(x) \\
|D|,|E| & \leq C U_{\rho}(x)
\end{aligned}
$$

and

$$
|B| \leq C U_{\rho}(x) \log \left|\frac{\rho}{\delta}\right| .
$$

Putting everything together, we have

$$
|\alpha(x)| \leq A_{\rho}(x)\left[1+\log \left(\frac{\rho}{\delta}\right)\right]+G_{\rho}(x) \delta \Omega_{\delta}(x) .
$$

where

$$
A_{\rho}(x)=C \rho^{-5 / 2}\left\|u_{0}\right\|_{L^{2}}+C \tilde{U} G_{\rho}(x)\left(1+\rho G_{\rho}(x)\right) .
$$


Note that by our assumptions, $\int_{0}^{T} A_{\rho} d t$ is bounded.

From this point on, our proof goes exactly as the one in Constantin-FeffermanMajda [13]. Therefore we omit the remaining of the proof here. However, we would like to explain intuitively why the boundedness of $\int_{0}^{T} A_{\rho} d t$ would guarantee the boundedness of $\omega$. If we can replace $\Omega_{\delta}(x)$ by $|\omega(x)|$, then by taking $\delta=|\omega(x)|^{-1}$, we will have

$$
D_{t}|\omega| \leq C A_{\rho}|\omega|[1+\log |\omega|]
$$

by which the boundedness of $|\omega|$ can be derived due to the boundedness of $\int_{0}^{T} A_{\rho}$. This will effectively end the proof. Also, at this point, it becomes clear why we need the assumption (3.28).

4. Conclusions. In this note, we explored a level set representation of vorticity in the study of the singularity problems for incompressible fluid models. This level set representation (also known as the Generalized Clebsch Variables representation) for the vorticity field involves three level set functions

$$
\omega=\sum_{i=1}^{3} \nabla U_{k} \times \nabla \phi_{k} .
$$

We showed that this representation exists for all incompressible flows. We further applied it to the singularity problems for the 3D Lagrangian averaged Euler equations and the 3D Euler equations, and obtained new global existence theorems for a large class of initial values, including all axisymmetric flows. Applications of this level set representation to singularity problems reveal new geometric structures of possible blow-up scenario.

\section{REFERENCES}

[1] A. Babin, A. Mahalov, B. Nicolaenko, 3D Navier-Stokes and Euler equations with initial data characterized by uniformly large vorticity, Ind. Univ. Math. Jour., 50 (2001), pp. 1-35.

[2] J. T. Beale, T. Kato, A. Majda, Remarks on the breakdown of smooth solutions for the 3-D Euler equations, Comm. Math. Phys., 94 (1984), pp. 61-66.

[3] T. F. Buttke, A. J. Chorin, Turbulence calculations in magnetization variables, Appl. Num. Math., 12:1-3 (1993), pp. 47-54.

[4] R. E. Caflisch, Singularity formation for complex solutions of the 3D incompressible Euler equations, Physica D., 67 (1993), pp. 1-18.

[5] S. Y. Chen, C. Foias, D. D. Holm, E. J. Olsen, E. S. Titi, S. Wynne, The Camassa-Holm equations as a closure model for turbulent channel and pipe flows, Phys. fluids, 11 (1999), pp. 2343-2353.

[6] S. Y. Chen, D. D. Holm, L. G. Margolin, R. Zhang, Direct numerical simulations of the Navier-Stokes alpha model, Physica D., 133 (1999), pp. 66-83.

[7] A. Clebsch, Uber die integration der hydrodynamischen gleichungen, J. Reine Angew. Math., 56 (1859), pp. 1.

[8] P. Constantin, Geometric statistics in turbulence, SIAM Rev., 36:1 (1994), pp. 73-98.

[9] P. Constantin, An Eulerian-Lagrangian approach for incompressible fluids: local theory, Journal of the AMS, 14 (2001), pp. 263-278.

[10] P. Constantin, An Eulerian-Lagrangian approach to the Navier-Stokes equations, Commun. Math. Phys., 216 (2001), pp. 663-686.

[11] P. Constantin, Near identity transformations for the Navier-Stokes equations, In Handbook of Mathematical Fluid Dynamics, Vol. 2, S. Friedlander and D. Serre Eds., Elsevier, 2003.

[12] P. Constantin, Euler Equations, Navier-Stokes Equations and Turbulence, Preprint, C. I. M. E. Lectures, 2004.

[13] P. Constantin, C. Fefferman, A. Majda, Geometric constraints on potentially singular solutions for the 3-D Euler equations, Comm. PDE, 21 (1996), pp. 559-571. 
[14] D. Cordoba, C. Fefferman, On the collapse of tubes carried by $3 D$ incompressible flows, Comm. Math. Phys., 222:2 (2001), pp. 293-298.

[15] J. Deng, T. Y. Hou, X. Yu, Geometric properties and nonblowup of $3 D$ incompressible Euler flow, Comm. PDE, 30:2 (2005), pp. 225-243.

[16] J. Deng, T. Y. Hou, X. Yu, Improved geometric conditions for nonblowup of the $3 D$ incompressible Euler equations, Comm. PDE, 31:2 (2006), pp. 293-306.

[17] C. R. Graham, F. S. Henyey, Clebsch representation near points where the vorticity vanishes, Physics of Fluids, 12:4 (2000), pp. 744-746.

[18] D. D. Holm, J. E. Marsden, T. S. Ratiu, Euler-Poincaré models of ideal fluids with nonlinear dispersion, Phys. Rev. Lett., 349 (1998), pp. 4173-4177.

[19] D. D. Holm, J. E. Marsden, T. S. Ratiu, Euler-Poincaré equations and semidirect products with applications to continuum theories, Adv. Math., 137 (1998), pp. 1-81.

[20] T. Y. Hou, C. LI, On Global Well-Posedness of the Lagrangian Averaged Euler Equations, to appear in SIAM J. Math. Anal.

[21] A. Majda, A. Bertozzi, Vorticity and Incompressible Flow, Cambridge University Press, 2002.

[22] J. E. Marsden, T. Ratiu, S. Shkoller, The geometry and analysis of the averaged Euler equations and a new diffeomorphism group, Geom. Funct. Analysis, 10 (2000), pp. 582599.

[23] J. E. Marsden, S. ShKoller, Global well-posedness for the lagrangian averaged Navier-Stokes (LANS- $\alpha$ ) equations on bounded domains, Phil. Trans. R. Soc. Lond. A, 359 (2001), pp. 1449-1468.

[24] J. E. Marsden, A. Weinstein, Coadjoint orbits, vortices, and Clebsch variables for incompressible fluids, Physica D, 7:1-3 (1983), pp. 305-323.

[25] S. Montgomery-Smith, M. Pokorný, A counterexample to the smoothness of the solution to an equation arising in fluid mechanics, Commentationes Mathematicae Universitatis Carolinae, 431 (2002), pp. 61-75.

[26] W. WeBER, Uber eine Transformation der hydrodynamischen Gleichungen, J. Reine Angew. Math., 68 (1868), pp. 286-292. 